[0212-7199 (2007) 24: 2; pp 87-97] ANALES DE MEDICINA INTERNA Copyright (C) 2007 ARAN EDICIONES, S.L.

AN. MED. InTERna (Madrid) Vol. 24, N. ${ }^{\circ} 2$, pp. 87-97, 2007

\section{La hormona paratiroidea en el tratamiento de la osteoporosis}

\author{
M. SOSA HENRÍQUEZ, A. DÍEZ PÉREZ
}

Universidad de Las Palmas de Gran Canaria. Grupo de Investigación en Osteoporosis. Servicio de Medicina Interna. Unidad Metabólica Ósea. Hospital Universitario Insular. Las Palmas de Gran Canaria. 'Servicio de Medicina Interna. Unidad de Investigación en Fisiopatología Ósea y Articular (URFOA). Hospital del Mar. Instituto Municipal de Investigación Médica (IMIM). Universidad Autónoma de Barcelona
PARATHYROID HORMONE IN THE TREATMENT OF OSTEOPOROSIS

\section{RESUMEN}

Todos los tratamientos hasta ahora disponibles para el tratamiento de la osteoporosis actuaban inhibiendo la resorción ósea y reduciendo el remodelado óseo. La parathormona intacta (PTH 1-84) y su análogo, la teriparatida (PTH recombinante humana 1-34), representan una nueva clase de tratamiento anabólico de la osteoporosis. Se ha descrito un efecto positivo en la mejoría de la microarquitectura ósea y una reducción en el riesgo de nuevas fracturas por un mecanismo osteoformador.

La PTH debe considerarse en el tratamiento de la osteoporosis grave, tanto en varones como en mujeres, en aquellos pacientes que tienen varias fracturas osteoporóticas o bien que presenten una densidad mineral ósea muy baja, (T-score inferior a $-3,5)$ y un elevado riesgo de fractura. Otros usos potenciales son en la osteoporosis inducida por corticoides u otras secundarias. No se recomienda su utilización durante más de 18 meses con teriparatida y con 24 meses con PTH 1-84.

PALABRAS CLAVE: PTH. Osteoporosis. Anabólico. Tratamiento. Fractura. Riesgo. Densidad mineral ósea. Postmenopausia. Mujeres.

\begin{abstract}
Current treatments available for osteoporosis until recently were active by inhibiting osteoclast activity and, thus, reducing bone remodelling. Intact PTH (PTH 1-84) and its analog, teriparatida (human recombinant PTH 1-34), are a new class of anabolic treatment of osteoporosis. It has been described a positive effect on bone microarchitecture and a reduction of the risk of new fractures due to a bone-forming mechanism.

PTH must be considered as an useful alternative in the treatment of severe osteoporosis, both in men and women, in patients with several osteoporosis-related fractures or with a very low bone mineral density (T-score below -3.5) an a high risk for fracture. Other potential uses are glucocorticoid-induced and other secondary osteoporosis. The use of PTH is not recommended for more than 18 months for teriparatida and 24 months for PTH 1-84.
\end{abstract}

KEY WORDS: PTH. Osteoporosis. Anabolic. Treatment. Fracture. Risk. Bone Mineral Density. Postmenopausal. Women.

Sosa Henríquez. M, Díez Pérez. A. La hormona paratiroidea en el tratamiento de la osteoporosis. An Med Interna (Madrid) 2007; $24: 87-97$.

\section{INTRODUCCIÓN}

La osteoporosis es la enfermedad metabólica ósea más frecuente en clínica humana. La definición aceptada por consenso es "enfermedad esquelética sistémica caracterizada por masa ósea baja y deterioro de la microarquitectura del tejido óseo, con el consiguiente aumento de la fragilidad del hueso y la susceptibilidad a fracturas" $(1,2)$. Los elementos esenciales de esta definición son la masa ósea baja y la alteración de la microarquitectura, que distinguen a la osteoporosis de otras enfermedades óseas. La alteración de la microarquitectura se caracteriza por la pérdida, adelgazamiento y falta de conexión entre trabéculas óseas, junto con una serie de factores, como alteraciones en el remodelado óseo, la propia geometría del hueso, etc., que se han agrupado bajo el concepto de calidad ósea (3). Todo ello produce un deterioro de la integridad estructural del hueso y favorece la fragilidad esquelética, lo cual conlleva un incremento del riesgo de fracturas. Precisamente las fracturas y sus complicaciones son las manifestaciones clínicas de la osteoporosis (4). Se consideran como típicamente osteoporóticas las fracturas de la extremidad proximal del fémur, vértebra y muñeca (5), aunque la mayor parte de los huesos son susceptibles de fracturarse (6).

En los últimos años hemos asistido a un amplio desarrollo de la tecnología y también se han publicado un buen número de estudios, realizados bajo los exigentes criterios de la medi- 
cina basada en la evidencia, que nos permiten utilizar una amplia cantidad de fármacos nuevos, con contrastada efectividad en la reducción del riesgo de fractura $(7,8)$. Por otra parte, la biología ha realizado grandes progresos en el conocimiento del ciclo de remodelado óseo, por lo que, en su conjunto, el panorama terapéutico existente hoy en día permite un manejo mucho mas racional y fisiopatológicamente más correcto de la osteoporosis.

En la presente revisión nos centraremos en la utilización en la osteoporosis, del fármaco genuinamente anabólico, la hormona paratiroidea (PTH), en sus dos presentaciones, la fracción 1-34 o teriparatida y la molécula intacta PTH 1-84.

\section{BASES FISIOPATOLÓGICAS DEL TRATAMIENTO DE LA} OSTEOPOROSIS CON PTH

Remodelación ósea es el término con que nos referimos a un constante proceso de renovación a que está sometido el hueso. Se lleva a cabo simultáneamente en múltiples unidades microscópicas bien delimitadas, dispersas por todo el esqueleto. En cada una de ellas el hueso es destruido y después sustituido por otro recién formado. Por medio de la remodelación ósea, el organismo sustituye el hueso envejecido o dañado por tejido nuevo y al mismo tiempo contribuye al mantenimiento de la homeostasis mineral (9).

En la osteoporosis se produce una alteración en el remodelado óseo. Por razones aún no completamente conocidas, se produce un disbalance entre la formación ósea, que realizan los osteoblastos y la resorción o destrucción de hueso, de la que es responsable los osteoclastos. En la osteoporosis postmenopáusica, existe típicamente un incremento de la resorción ósea, manteniéndose la formación normal o discretamente disminuída $(10,11)$. Como consecuencia de ello, se produce un balance negativo que conduce a la pérdida de masa ósea. Precisamente la baja densidad mineral ósea es el factor de riesgo más importante para la fractura osteoporótica $(12,13)$.
En la fisiopatología de la osteoporosis, existe también un factor cuantitativo, que es la alteración de la microarquitectura a que da lugar el aumento del recambio óseo, produciendo inestabilidad del esqueleto, microperforaciones y microfracturas (14-15). Aún más, en prácticamente todos los estudios clínicos en los que se han utilizado fármacos antirresortivos, como la calcitonina, estrógenos, inhibidores selectivos de los receptores estrogénicos (SERMs) y bisfosfonatos, se ha observado que la reducción del riesgo de fractura no guarda ninguna proporción con el incremento en la densidad mineral ósea $(16,17)$.

Por otra parte, en enfermedades en las que existe un exceso de PTH, como en el hiperparatiroidismo primario (HPTP) se ha podido constatar el efecto anabólico de la PTH. Así, en el estudio de Silverberg y cols. (18) se comprobó que las formas asintomáticas de HPTP se produce una conservación del hueso trabecular, que llega a ser cualitativamente de mejor calidad que el hueso de los controles de su misma edad y sexo $(19,20)$.

Teniendo en cuenta estos hechos, es fácil comprender que el tratamiento de la osteoporosis con PTH implica una aproximación diferente a las terapias habitualmente utilizadas hasta el momento. La PTH actúa directamente sobre los osteoblastos, dado que estas células formadoras de hueso tienen receptores específicos para esta hormona (21), produciendo formación de hueso por un mecanismo doble: por una parte, por el incremento del índice de remodelado óseo y por otra al obtener un balance positivo de la cantidad de hueso depositada en cada unidad de remodelamiento, tal y como se comprueba por biopsia, al aumentar el grosor trabecular en las osteonas (22). Esto diferencia el efecto del tratamiento con PTH de otras formas clínicas de alto remodelado, como ocurre con la deficiencia estrogénica, que tiene un efecto negativo sobre el hueso. El resultado es la producción directa de nuevo hueso con la consecuente ganancia de densidad mineral ósea y la reducción del riesgo de fractura. Todos estos detalles los comentaremos y documentaremos a continuación.

\section{TABLA I}

REDUCCIÓN DEL RIESGO DE FRACTURA VERTEBRAL CON PTH

\begin{tabular}{|c|c|c|c|c|c|}
\hline Primer autor & Cita & Fármaco & $\begin{array}{l}\text { Grupo de pacientes tratados } \\
N^{\circ} \text { con fracturas } / N^{a} \text { total }\end{array}$ & $\begin{array}{l}\text { Grupo de pacientes control } \\
N^{0} \text { con fracturas } / N^{a} \text { total }\end{array}$ & Odds Ratio IC 95\% \\
\hline Lane & 72 & Teriparatida & $0 / 26$ & $1 / 18$ & $0,23(0,01-5,45)$ \\
\hline Cosman & 43 & Teriparatida & $0 / 25$ & $7 / 27$ & $0,07(0,00-1,20)$ \\
\hline $\begin{array}{l}\text { Neer } \\
20 \_g \\
40 \text { mg } \\
\end{array}$ & 28 & Teriparatida & $\begin{array}{l}19 / 434 \\
22 / 444\end{array}$ & $64 / 448$ & $\begin{array}{l}0,31(0,19-0,50) \\
0,35(0,22-0,55)\end{array}$ \\
\hline $\begin{array}{l}\text { Cosman } \\
\text { Diaria } \\
\text { Intermitente }\end{array}$ & 43 & Teriparatida & $\begin{array}{l}1 / 38 \\
2 / 34\end{array}$ & $4 / 36$ & $\begin{array}{l}0,24(0,03-2,02) \\
0,53(0,10-2,71)\end{array}$ \\
\hline $\begin{array}{l}\text { Orwoll } \\
20 \mu g \\
40 \mu g\end{array}$ & 67 & Teriparatida & $\begin{array}{l}5 / 92 \\
5 / 84\end{array}$ & $12 / 103$ & $\begin{array}{l}0,47(0,17-1,27) \\
0,51(0,19-1,39)\end{array}$ \\
\hline Kurland & 66 & Teriparatida & $1 / 6$ & $2 / 12$ & $1,00(0,11-8,95)$ \\
\hline Greenspan & 47 & PTH 1-84 & $17 / 1,286$ & $42 / 1,246$ & $0,39(0,22-0,69)$ \\
\hline Vestergaard & 98 & Metanálisis & $72 / 2,469$ & $132 / 1,890$ & $0,36(0,28-0,47)$ \\
\hline
\end{tabular}




\section{VISIÓN HISTÓRICA DEL TRATAMIENTO CON PTH}

En realidad, el tratamiento de la osteoporosis con PTH no es nuevo. Ya hace más de 30 años, en un estudio preliminar, Reeve y col publicaron por primera vez en la década de los 70 una serie de 4 pacientes a las que se le administró PTH, (fragmento 1-34) a diferentes dosis (entre 100 y 400 $\mu \mathrm{g} /$ día en ciclos de 8 días). Como parámetro para valorar la efectividad del tratamiento se utilizó el balance de calcio, que se volvió positivo en todos los casos, llegando los autores a calcular la cantidad del mineral depositado en el esqueleto (23). En esa época no se disponía aún de densitometría. El mismo grupo de trabajo presentó algunas publicaciones, como seguimiento de estos mismos pacientes $(24,25)$. Pese a los resultados prometedores, este grupo de trabajo abandonó esta línea de investigación. Casi una década después, Slovik y cols. (26) presentaron una serie de 8 pacientes tratados con PTH durante 12 meses y en los que se obtuvo un incremento en la densidad mineral ósea (DMO) determinada por tomografía axial computadorizada (QCT). Hubo también otra iniciativa terapéutica con la PTH, con el llamado tratamiento secuencial o ADFR (Activar, Deprimir, Periodo libre -Free- y Repetir) propuesto por Frost (27). La fase de activación se realizaba con fósforo y buscaba indirectamente la liberación de PTH endógena. Este tratamiento fue también abandonado.

\section{LA PTH 1-34 (TERIPARATIDA)}

Con este nombre se conoce a la PTH que contiene los primeros 34 aminoácidos de la PTH humana, donde radica el segmento metabólicamente activo de la hormona, producida por tecnología de ADN recombinante, Por razones de espacio y para no desviarnos de la línea original de esta revisión clínica, no comentaremos en esta revisión los estudios efectuados en animales de experimentación.

\section{ESTUDIOS DE PTH COMO MONOTERAPIA}

El estudio pivotal con teriparatida es el "Fracture Prevention Trial”, realizado en 1637 mujeres postmenopáusicas con DMO baja y al menos una fractura prevalente y que no recibían ni tratamiento hormonal sustitutivo ni ningún otro tratamiento antirresortivo (28). Fueron agrupadas de manera aleatoria en 3 grupos que recibieron 20 ó $40 \mu \mathrm{g} /$ día de teriparatida o placebo. Aunque el estudio estaba inicialmente planificado para tener una duración de 36 meses, fue suspendido cuando las pacientes llevaban una media de 21 meses de tratamiento, debido a la publicación de casos de osteosarcomas en ratas Fischer que llevaban un tiempo muy prolongado recibiendo el fármaco a dosis muy elevadas. Posteriores estudios de toxicidad demostraron un perfil de seguridad positivo que permitió proseguir su desarrollo clínico.

Las pacientes que recibieron teriparatida presentaron un incremento en la DMO de la columna lumbar del $9 \%$ con 20 $\mu \mathrm{g} /$ día y del $13 \%$ con $40 \mu \mathrm{g} /$ día, así como un incremento en el cuello femoral del $3 \%$ con $20 \mu \mathrm{g} /$ día y del $6 \%$ con $40 \mu \mathrm{g} /$ día. En el radio, la DMO descendió en los 3 grupos estudio (los dos que recibieron teriparatida y los controles), siendo estadísticamente significativo el descenso en el grupo que recibió $40 \mu \mathrm{g} /$ día con respecto al grupo placebo. Comparados con el grupo placebo. el riesgo de desarrollar una nueva fractura vertebral disminuyó en un $65 \%$ en el grupo que recibió $20 \mu \mathrm{g} / \mathrm{día}$ y un $69 \%$ en el grupo de $40 \mu \mathrm{g}$ /día $(29,30)$. El riesgo de fracturas no vertebrales disminuyó un $53 \%$ en el grupo que recibió $20 \mu \mathrm{g} /$ día y un $54 \%$ en el grupo de $40 \mu \mathrm{g} /$ día, también comparado con el grupo placebo. Por otra parte, otras publicaciones derivadas de este mismo estudio mostraron que la teriparatida disminuía el riesgo de nuevas FV y FNV en aquellas pacientes que por presentan fracturas previas más numerosas y/o más graves tenían más riesgo de nuevas fracturas (31). También se ha comprobado que la pérdida de estatura fue significativamente menor en los 2 grupos que habían recibido teriparatida frente al placebo (28), que el dolor de espalda disminuyó de manera también estadísticamente significativa

TABLA II

REDUCCIÓN DEL RIESGO DE FRACTURA NO VERTEBRAL CON PTH

\begin{tabular}{|c|c|c|c|c|c|}
\hline Primer autor & Cita & Fármaco & $\begin{array}{l}\text { Grupo de pacientes tratados } \\
N^{\circ} \text { con fracturas } / N^{a} \text { total }\end{array}$ & $\begin{array}{l}\text { Grupo de pacientes control } \\
N^{0} \text { con fracturas } / N^{a} \text { total }\end{array}$ & Odds Ratio IC 95\% \\
\hline Lane & 72 & Teriparatida & $2 / 26$ & $2 / 23$ & $0,88(0,14-5,79)$ \\
\hline $\begin{array}{l}\text { Neer } \\
\qquad \begin{array}{l}20 \mu \mathrm{g} \\
40 \mu \mathrm{g}\end{array}\end{array}$ & 28 & Teriparatida & $\begin{array}{l}34 / 541 \\
32 / 552\end{array}$ & $53 / 544$ & $\begin{array}{l}0,65(0,43-0,98) \\
0,60(0,39-0,91)\end{array}$ \\
\hline $\begin{array}{l}\text { Cosman } \\
\text { Diaria } \\
\text { Intermitente }\end{array}$ & 43 & Teriparatida & $\begin{array}{l}4 / 38 \\
2 / 34\end{array}$ & $2 / 36$ & $\begin{array}{l}1,89(0,37-9,72) \\
1,06(0,16-7,10)\end{array}$ \\
\hline $\begin{array}{l}\text { Orwoll } \\
20 \mu g \\
40 \mu g\end{array}$ & 67 & Teriparatida & $\begin{array}{l}2 / 151 \\
1 / 139\end{array}$ & $3 / 147$ & $\begin{array}{l}0,65(0,11-3,83) \\
0,34(0,04-3,35)\end{array}$ \\
\hline Body & 36 & Teriparatida & $3 / 73$ & $10 / 73$ & $0,30(0,09-1,05)$ \\
\hline Vestergaard & 98 & Metanálisis & $80 / 1,554$ & $70 / 823$ & $0,62(0,48-0,82)$ \\
\hline
\end{tabular}


entre las pacientes tratadas, frente a las controles $(30,32)$ y un efecto beneficioso de la teriparatida sobre la calidad de vida en las pacientes afectas de fracturas vertebrales $(33,34)$.

Un amplio número de estudios efectuados tanto con marcadores bioquímicos de remodelado óseo como con biopsias óseas, han demostrado que la teriparatida tiene un marcado efecto anabólico sobre el hueso, estimulando la actividad osteoblástica (35-37) y produciendo también mejoría tanto de la microarquitectura trabecular como de otros aspectos del hueso, que globalmente se han denominado "calidad ósea" (38-40). Entre ellos cabe destacar la acción positiva sobre la geometría del radio distal que aumenta su resistencia a pesar de la aparente disminución de la densidad ósea (41).

\section{ESTUDIOS DE PTH 1-34 EN COMBINACIÓN CON OTROS FÁRMACOS}

Algunos estudios indican que la utilización concomitante de PTH 1-34 con antirresortivos tiene efectos diversos sobre el efecto anabólico de la hormona al principio del tratamiento $(42,43)$. Así, se ha estudiado el efecto de la teriparatida a dosis diaria de $400 \mathrm{U}(25 \mu \mathrm{g})$ en 52 mujeres postmenopáusicas con osteoporosis que ya venían recibiendo THS al menos durante 2 años. Fueron aleatorizadas para recibir teriparatida o placebo durante 36 meses. (43). Las pacientes que recibieron PTH y THS mostraron un incremento en la DMO del 13,4, 4,4 y $3,7 \%$ en la columna lumbar, total de cadera y cuerpo total respectivamente, mientras que no se apreciaron diferencias estadísticamente significativas en aquellas que se mantuvieron sólo con THS. La naturaleza del estudio y el tamaño muestral no permitió confirmar la reducción en el riesgo de fractura vertebral pero lo que parece indicar es que el efecto de ambos fármacos se potencia. Un efecto similar se ha descrito al combinar teriparatida y raloxifeno (104).

Por el contrario, otro estudio aleatorizado, pero realizado en 83 varones afectos de osteoporosis, comparó el efecto sobre el hueso de alendronato, a dosis de $10 \mathrm{mg} /$ día, PTH 134, $40 \mu \mathrm{g}$ /día, y una combinación de ambos (42). El alendronato se administró durante 30 meses y el tratamiento con PTH comenzó a los 6 meses. Al finalizar el estudio, se observó un incremento estadísticamente significativo en la DMO de la columna lumbar determinada tanto por DXA como por QCT del grupo que recibió sólo PTH en comparación con el que recibió la combinación de PTH y alendronato. Además, la DMO en el cuello femoral fue mayor a los 30 meses en el grupo que recibió sólo PTH que en el otro, cuando a los 18 meses, la DMO en el cuello femoral era inferior.

Estos resultados sugieren que la administración conjunta de alendronato y teriparatida reduce el efecto anabólico de la PTH en el hueso trabecular de la columna y altera también la capacidad que tiene la PTH de aumentar el volumen cortical del fémur proximal durante los primeros meses de tratamiento. En lo que respecta a la DMO del cuello femoral, la combinación de teriparatida y alendronato parece superior a la teriparatida sola, pero sólo a corto plazo, 18 meses, porque a largo plazo la teriparatida produce mayores beneficios. Por lo tanto, la administración simultánea de PTH y alendronato no puede recomendarse $(44,45)$ cosa que parece no ocurrir al combinarla con antirresortivos menos inhibitorios del remodelado como la terapia hormonal o los SERMs.

\section{LA PTH MOLÉCULA INTACTA O PTH 1-84}

\section{ESTUDIOS CON PTH COMO MONOTERAPIA}

Hodsman y cols. (46) realizaron un estudio en 217 mujeres postmenopáusicas afectas de osteoporosis, con una edad media de 64.5 años, a las que se les administró de forma aleatorizada placebo o PTH 1-84 a dosis de 50, 75, o $100 \mu \mathrm{g}$ ). El objetivo principal fue cambios en la densidad mineral ósea de la columna lumbar y el estudio se prolongó durante 1 año.

Al finalizar el estudio, la media de incremento en la DMO fue del 3,0, 5,1 y 7,8\% en los grupos cuya dosis fue respectivamente 50-, 75- y 100- $\mu \mathrm{g} / \mathrm{dí}$, siendo todos los incrementos estadísticamente significativos y claramente dosis-dependiente, mientras que en el grupo control, que recibió calcio y vitamina $\mathrm{D}$, se observó un aumento del $0.9 \%$ que no alcanzó significación estadística. El aumento en la DMO obtenida por el grupo que recibió $100 \mu \mathrm{g}$ fue estadísticamente significativo con respecto a los otros dos grupos que recibieron PTH, pasando el T-score de -3,2 al inicio del estudio a -2.8 al finalizar el mismo. Por el contrario no se apreciaron diferencias estadísticamente significativas en la DMO de la cadera.

El estudio TOP (Treatment of Osteoporosis), es un trabajo efectuado durante 18 meses con 2.532 mujeres postmenopáusicas afectas de osteoporosis siendo el objetivo principal la reducción del riesgo de fractura vertebral (47). Entre los criterios de inclusión se exigió una edad superior a 55 años, con un valor densitométrico inferior a -2,5 en columna lumbar o cadera o inferior a -2 con al menos una fractura vertebral. Aquellas más jóvenes de entre 45-55 años, debían mostrar en la densitometría un valor inferior a -3 en un grupo sin fracturas o inferior a $-2,5$ pero que tuviesen al menos una fractura vertebral. En su conjunto, la edad media de las pacientes que participaron en el estudio fue de 64 años y, de ellas, el 19\% tenía al menos una fractura vertebral. A los 18 meses, el incremento observado en la densidad mineral ósea de la columna lumbar de las mujeres tratadas con PTH intacta (1-84) fue del 7\% en comparación con el grupo placebo. El riesgo de nueva fractura vertebral descendió en un 66\% en el grupo que recibió tratamiento con PTH y este descenso se observó tanto en las mujeres que previamente tenían al menos una fractura vertebral como en las que no. El 28,3\% de las mujeres tratadas presentaron hipercalcemia, frente al 4,7\% del grupo control (47).

La tasa más elevada de hipercalcemias en este estudio, comparada con los datos publicados de otros estudios, como el PaTH en el que la incidencia de este efecto adverso se redujo al $12 \%$, se justifica por parte de los autores por el hecho de que no se realizaron determinaciones de PTH ni vitamina D en los pacientes previas a la inclusión en el estudio, y se permitió la inclusión de pacientes con hipercalcemia moderada (entre 10,2 y 10,7 mg/dl). Este hecho proporcionó una población de pacientes más similar a la que presenta osteoporosis, lo que hace que los resultados del estudio pudiesen ser más generalizables. Sin embargo, la decisión de restringir la inclusión de pacientes con posible hiperpatratiroidismo tomada en otros estudios con osteoformadores pudo haber reducido el número de casos de hipercalcemia (47).

\section{ESTUDIOS DE PTH 1-84 EN COMBINACIÓN CON OTROS FÁRMACOS}

Black y cols. (49) publicaron un trabajo realizado en 238 mujeres afectas de osteoporosis postmenopáusica, el deno- 
minado estudio PaTH, a las que se les asignó de manera aleatorizada $100 \mu \mathrm{g} /$ día de PTH 1-84, $(\mathrm{n}=119)$, alendronato, $10 \mathrm{mg} /$ día $(\mathrm{n}=60)$ o ambos fármacos conjuntamente $(\mathrm{n}=$ 59). El estudio se prolongó durante 1 año, siendo el objetivo principal del estudio fue analizar si la combinación de 2 fármacos era superior a cualquiera de los mismos por separado. Se determinó la DMO por densitometría en la columna lumbar y en la cadera y por tomografía axial computadorizada (QCT) y también se estimaron los marcadores bioquímicos de remodelamiento óseo. La DMO aumentó en la columna lumbar en todos los grupos tratados, pero no se apreciaron diferencias estadísticamente significativas entre el incremento observado entre el grupo que recibió PTH sola y el que recibió la combinación de PTH y alendronato. La densidad volumétrica del hueso trabecular de la columna lumbar, determinada por QCT aumentó sustancialmente en todos los grupos, pero en las mujeres que recibieron PTH el aumento fue el doble del obtenido en los otros grupos. La formación ósea, valorada por los marcadores bioquímicos de remodelado óseo, aumentó en el grupo tratado con PTH, pero no fue así en el grupo que recibió PTH y alendronato, lo cual probablemente sugiere que el uso simultáneo de este antirresortivo reduce el efecto anabólico de la PTH, al menos durante los primeros meses de tratamiento (45). En otra publicación de este mismo estudio, Bauer y cols. (50) demostraron que aquellas pacientes que presentaron un mayor incremento en los marcadores de remodelado óseo en los 3 primeros meses, (especialmente los marcadores de formación) posteriormente al cabo de 1 año mostraron un mayor incremento de la densidad mineral ósea.

Después de la aparición del PaTH, se han publicado resultados con determinados subgrupos de pacientes analizando otros objetivos secundarios. Así por ejemplo, Fox y cols. (51) realizaron biopsias óseas a 8 pacientes que recibieron PTH (184) y a 8 controles, obteniendo un importante incremento, estadísticamente significativo, en la mayor parte de los parámetros histomorfométricos estudiados, mientras que Antoniucci y cols. (52), estudiaron al subgrupo de 178 mujeres que recibieron PTH sola o en combinación con alendronato, es decir excluyendo a las que recibían sólo alendronato, encontrando una prevalencia de hipercalcemia del $14 \%$ y una prevalencia de hipercalciuria del $8 \%$; en el $88 \%$ de estos casos, éstas desaparecieron espontáneamente o tras suspender el calcio o la vitamina $\mathrm{D}$.

En una publicación canadiense, Rittmaster y cols. (53) estudiaron a un grupo de 66 mujeres que habían recibido PTH $1-84$ a dosis de 50, 75 y $100 \mu \mathrm{g}$ /día durante 1 año y tras suspender ésta, les administraron a continuación $10 \mathrm{mg} /$ día de alendronato durante 1 año más. Durante el primer año, la DMO en el total de las mujeres (incluyendo todas las diferentes dosis de PTH) aumentó un 7,1 $\pm 5,6 \%$ en la columna lumbar, un $0,3 \pm 6,2 \%$ en el cuello femoral y un $22,3 \pm 3,3 \%$ en todo el cuerpo. Tras pasar a alendronato, al cabo de 1 año los cambios en la densidad mineral ósea fueron del 13,4 $\pm 6,4 \%$ en la columna lumbar, $4,4 \pm 7,2 \%$ en el cuello femoral y del $2,6 \pm 3,1 \%$ en todo el cuerpo. En el subgrupo de pacientes que recibieron las dosis más elevadas de $\mathrm{PTH}$, el incremento medio de DMO en la columna lumbar fue del 14,6 $\pm 7,9 \%$. Mientras se mantuvo el tratamiento con PTH, los marcadores bioquímicos de remodelado óseo permanecieron aumentados y descendieron por debajo del valor inicial después de 1 año con alendronato.

\section{LA PTHrP}

La PTHrP (péptido relacionado con la PTH) es una proteína que se identificó como la causante de la hipercalcemia tumoral y que se aisló a partir de tumores (54-57) y que es muy similar a la PTH 1-34 (58-60). Como consecuencia de ello, esta proteína se une activando al receptor PTHR1 que es el responsable de mediar tanto en los efectos paracrinos pleiotrópicos de la PTHRP como en los efectos endocrinos de la PTH en la homeostasis del calcio y del esqueleto (61).

Los osteoblastos tienen el receptor PTHR1 y por ello, tanto la PTH como la PTHrP ejercen su efecto anabólico directamente sobre ellos $(62,63)$. Sin embargo, sus efectos son algo diferentes. Así, en un estudio realizado para investigar cuál es el mecanismo fisiopatológico por el que ambas producen hipercalcemia, se observó que la PTH es más selectiva en estimular la síntesis renal de 1,25 dihidroxivitamina D, mientras que la PTHrP produce una mayor reabsorción tubular de calcio (64) Ambas moléculas comparten el efecto anabólico sobre el osteoblasto y por ello, recientemente se han publicado algunos estudios que sugieren que la PTHrP podría tener un papel en el tratamiento de la osteoporosis, dadas sus propiedades anabólicas sobre el hueso. Así, Horwitz y cols. (65) realizaron un estudio prospectivo, doble-ciego, caso-control en 16 mujeres postmenopáusicas afectas de osteoporosis. Todas recibieron calcio y vitamina D y tratamiento hormonal sustitutivo. A un grupo se le administró $400 \mu \mathrm{g} /$ día de PTHrP vía subcutánea y el otro recibió placebo. En el grupo tratado se observó un incremento de la DMO en la columna lumbar del $4,7 \%$, con aumento de la osteocalcina sérica en el corto período de 3 meses. Pese a las elevadas dosis utilizadas, la tolerancia al fármaco fue buena. Aún no disponemos de datos suficientes y son necesarios más estudios, con tamaños muestrales mayores y con el objetivo primario de reducción del riesgo de fractura, para recomendar la utilización de la PTHrP en el tratamiento de la osteoporosis.

\section{ADMINISTRACIÓN DE UN ANTIRRESORTIVO DESPUÉS DE LA PTH}

Ya desde la realización de estudios en animales comenzó a observarse que la administración de una tratamiento antirresortivo después de la PTH tenía un efecto beneficioso sobre la DMO. En una extensión abierta de un estudio previamente aleatorizado, se administró alendronato a dosis de $10 \mathrm{mg} /$ día a mujeres postmenopáusicas afectas de osteoporosis que previamente habían recibido $\mathrm{PTH} 1-84$ (50, 75, o $100 \mu \mathrm{g} /$ día) o placebo durante 1 año (53). Se observó un incremento estadísticamente significativo de la DMO en la columna lumbar tras el primer año de tratamiento que fue estadísticamente significativo en las pacientes que habían recibido las dosis más elevadas de PTH. Sin embargo, no se apreciaron cambios en la DMO del cuello femoral en ninguno de los 3 grupos que recibieron tratamiento, comparados con el placebo, e incluso se observó un descenso en la DMO de todo el cuerpo en los 2 grupos que recibieron más dosis de PTH, pero no en las que recibieron $50 \mu \mathrm{g} /$ día ni en el placebo. Después de 1 año de tratamiento con alendronato, se produjo un incremento adicional en la DMO de la columna lumbar del 5,7, 6,3, 6,2 y del 4,9\% en el grupo placebo. En el cuello femoral, los incrementos obtenidos fueron del $4,2,5,5$ y $2,8 \%$, y del $4,5 \%$ en el grupo control. En la DMO 
de todo el cuerpo, también se obtuvo un discreto pero significativo incremento en la $\operatorname{DMO}(2,6,3,2,5,2$, y $6,1 \%$, respectivamente), Por lo tanto, la administración de alendronato después del tratamiento con PTH produce un incremento estadísticamente significativo de la DMO en la columna lumbar y además revierte el efecto neutral o negativo de la PTH en el cuello femoral y en todo el cuerpo.

En la continuación del estudio secuencial PaTH que comparaba PTH (1-84) con alendronato y la asociación de PTH (1-84) con alendronato, al cabo de una año de tratamiento, se clasificó de manera aleatoria a los pacientes que habían recibido PTH (1-84) a recibir de forma ciega alendronato o placebo. La densidad mineral ósea de la columna lumbar aumentó significativamente ( $\mathrm{p}<0,001)$, siendo el aumento más importante el correspondiente al grupo que recibió PTH (1-84) seguido de alendronato $(12,1 \%)$ frente al que recibió PTH (1-84) seguido de placebo (4,1\%; diferencia de un $8 \%$; IC 95\%). En el cuello femoral y la cadera total, hubo aumentos significati$\operatorname{vos}(\mathrm{p}<0,001)$ en la densidad mineral ósea que fueron más importantes en el grupo de PTH (1-84) seguido de alendronato frente al grupo de PTH (1-84) seguido de ( $\mathrm{p}=0,005$ para el grupo de cuello femoral y $\mathrm{p}<0,001$ para el de cadera total). La densidad mineral volumétrica en el hueso trabecular medida por QCT aumentó en la columna lumbar y en la cadera en todos los grupos del estudio, salvo en el grupo que recibió alendronato durante 24 meses $\mathrm{p}=0,06$ ). Los aumentos de densidad mineral volumétrica en el hueso trabecular de la columna fueron mayores en el grupo que recibió PTH (1-84) seguida de alendronato) (31\% p < 0,001). Estos resultados sugieren el administrar un antirresortivo después de concluir el tratamiento con PTH (107).

\section{LA PTH EN EL TRATAMIENTO DE LA OSTEOPOROSIS EN EL} VARÓN

\section{ESTUDIOS CON TERIPARATIDA}

Además del estudio inicial de Slovik y cols. (25) que podríamos considerar casi anecdótico por el reducido tamaño muestral, se han publicado otros estudios, metodológicamente más completos, que permiten establecer la utilidad de la PTH en el tratamiento de la osteoporosis en el varón.

El primer estudio de estas características fue el realizado por Kurland y cols. (66) en el que participaron 23 varones que recibieron 400 unidades día de teriparatida (equivalentes a 25 $\mu \mathrm{g} /$ día) o placebo durante 18 meses. Los pacientes que recibieron el fármaco presentaron un incremento del 13,5\% en la DMO de la columna lumbar. La DMO de la cadera también aumentó, pero en menor cantidad $(2.9 \%)$ y más lentamente, mientras que la DMO en el radio no se modificó significativamente. En otro estudio realizado con 437 pacientes con osteoporosis idiopática o por hipogonadismo, Orwoll y cols. (67) administraron 20 ó $40 \mu \mathrm{g}$ /día de teriparatida al grupo tratado y calcio y vitamina $\mathrm{D}$ al grupo placebo, obteniendo en los tratados un incremento del 5,9\% en la columna lumbar y del 1,5\% en el cuello femoral. Este estudio fue detenido a los 11 meses y no se estudió la incidencia de nuevas fracturas. Posteriormente se efectuó un estudio "posthoc" para analizar la reducción del riesgo de fracturas, que se comenta en el apartado siguiente.

\section{ESTUDIOS CON PTH INTACTA Y CON PTHRP}

No disponemos en la actualidad estudios con PTH intacta ni con PTHrP en el tratamiento de la osteoporosis en el varón.

\section{¿QUÉ OCURRE CUANDO SE SUSPENDE EL TRATAMIENTO CON PTH?}

Cuando el tratamiento se suspende en los varones se observan resultados similares. En el estudio de Owoll y cols. (67) se efectuó el seguimiento de una rama de 279 pacientes durante 30 meses (18 meses después de suspender el tratamiento), los cuales disponían de radiografía lateral de tórax al inicio de estudio. En el grupo de pacientes que habían recibido teriparatida (20 ó $40 \mu \mathrm{g} /$ día), el riesgo de fractura vertebral descendió un $51 \%$, pero no alcanzó significación estadística $(\mathrm{p}=0,07)$. Sin embargo, cuando se consideraron sólo las fracturas moderadas o severas y se comparó al grupo tratado con el grupo placebo, si se alcanzó significación estadística $(6,8$ vs. $1,1 \% ; \mathrm{p}<0,02)(68)$.

En las mujeres se observa exactamente lo mismo. Lindsay y cols. (69) realizaron un estudio de seguimiento del trabajo de Neer y cols. (28) y describieron que el riesgo de fractura vertebral permanecía más bajo en aquellas mujeres que habían sido previamente tratadas con teriparatida en comparación con los controles que recibieron sólo placebo. A los 18 meses de suspender la teriparatida o el placebo, la incidencia de fractura vertebral observada fue del $11,3 \%$ en las mujeres del grupo que recibió $20 \mu \mathrm{g} /$ día, del 10,4\% en el grupo de $40 \mu \mathrm{g} / \mathrm{día}$ y del $19 \%$ en las mujeres del grupo placebo. La reducción del riesgo fue más significativa en las mujeres tratadas con teriparatida que tenían una osteoporosis más grave y en ellas se observó también una disminución del dolor de espalda y unos valores de DMO superiores a los controles tras 18 meses sin tratamiento.

\section{LA PTH EN EL TRATAMIENTO DE LA OSTEOPOROSIS INDUCIDA} POR ESTEROIDES

Existen pocos estudios efectuados en pacientes afectas de osteoporosis esteroidea. Un trabajo realizado por Rehman y cols. (70), en mujeres afectas de osteoporosis por corticoides, administró teriparatida a un grupo de 28 mujeres que ya recibían tratamiento hormonal sustitutivo y mantuvo sólo el tratamiento hormonal sustitutivo al grupo control, formado por 23 mujeres, también afectas de osteoporosis. Al cabo de 1 año se observó un incremento estadísticamente significativo en el área vertebral transversal de L1 y L2, estimada por tomografía axial computadorizada (QCT) del 4,8\% (p < 0,001). Además, 1 año después de suspender la teriparatida, se observó un incremento del $2,6 \%$ con respecto a los valores basales $(p<$ $0,05)$, mientras que en el grupo control no se apreciaron diferencias estadísticamente significativas. En otro estudio publicado por Hurley y cols. (71) estudiaron a 51 mujeres que recibían corticoides de manera crónica, a una dosis media de entre 5-20 mg/día de prednisona. Veintiocho mujeres recibieron teriparatida a dosis de $40 \mu \mathrm{g} /$ día y continuaron con el Tratamiento hormonal sustitutivo, mientras que 23 mujeres que sólo recibieron tratamiento hormonal sustitutivo constituyeron el grupo control. En todos los casos se administró calcio y vitamina D. 
Lane y cols. (72,73) realizaron un estudio en 51 mujeres postmenopáusicas que ya recibían esteroides de manera crónica por una amplia variedad de enfermedades inflamatorias crónicas y tratamiento hormonal sustitutivo. Aleatoriamente las agrupó para que 28 mujeres recibieran además teriparatida, mientras que 23 recibieron placebo. El estudio se prolongó durante 1 año y se observó en el grupo tratado un incremento de la DMO vertebral de casi un $35 \%$, determinada por QCT y un incremento adicional del 2,9\% tras 12 meses de seguimiento sin PTH. En el grupo control, el incremento en la DMO tras 12 meses de tratamiento fue del $1,3 \%$ y tras un año más de seguimiento se observó un incremento adicional del 0,3\%.

No disponemos de estudios realizados con PTH intacta o con PTH-RP sobre este particular tipo de osteoporosis secundaria.

\section{SEGURIDAD DEL TRATAMIENTO CON PTH}

La PTH es bien tolerada (74). Los efectos secundarios de la teriparatida, recogidos en la serie original de 1943 pacientes de Neer y cols. (28) incluyen náuseas, cefaleas y mareos que aparecieron en las pacientes que recibieron las dosis más elevadas de teriparatida. También se observó hipercalcemia leve, definida como una concentración de calcio sérico superior a $10,6 \mathrm{mg} / \mathrm{dl}$, que se produjo en el $2 \%$ de las mujeres que recibieron placebo, en el $11 \%$ de las pacientes del grupo de $20 \mathrm{mg}$ de teriparatida y en el $28 \%$ entre aquellas del grupo que recibió $40 \mu \mathrm{g} /$ día. En todos los casos la hipercalcemia fue transitoria y no se requiere monitorización de la calcemia en el tratamiento con teriparatida. En el caso de la PTH 1-84 si que se requiere monitorización de los niveles de calcio, especialmente al principio de los estudios, en base a los datos de los ensayos, ya que el $24 \%$ de los pacientes del estudio TOP presentaron hipercalciuria y el $23 \%$ hipercalcemia (48). Sin embargo, el número de discontinuaciones del tratamiento por esta causa fue escaso en los ensayos clínicos publicados (dos pacientes en el estudio PaTH y seis pacientes en el estudio TOP) y generalmente el efecto se controla retirando los suplementos de calcio y vitamina $\mathrm{D}$ que reciben los pacientes sin precisarse la reducción de la dosis o la retirada del tratamiento (47).

El efecto tóxico que se ha observado sólo en animales, el osteosarcoma, es el mismo se produce con una dosis de teriparatida o PTH intacta a dosis muy elevadas y durante unos períodos de tiempo equivalentes a varias décadas de tratamiento en humanos, que no son equiparables a las administradas en humanos (75-78) (Tabla III). Este periodo de tiempo incluye una situación ósea de crecimiento por lo que no está indicado el uso de la PTH en niños o adolescentes, antes del cierre epifisario. De hecho, las dosis con la que han aparecido osteosarcomas en ratas son de 20 a 58 veces superiores a las indicadas terapéuticamente. En una revisión, Tashjian y Gagel informan que hasta agosto de 2005 no se había recogido ni un sólo caso de osteosarcoma en humanos, después de la prescripción de más de 250.000 tratamientos con teriparatida o con PTH intacta (79) y tampoco después del seguimiento de los pacientes que participaron en los estudios tanto con teriparatida como con PTH intacta en la década de los 80. Más recientemente se ha comunicado un caso de osteosarcoma en un paciente tratado con PTH 1-34 (105) pero la incidencia representa la misma o algo menor que la basal de la población general no tratada por lo que no representa señal de incremen-
TABLA III

INCIDENCIA DE OSTEOSARCOMAS EN RATAS TRATADAS

TANTO CON TERIPARATIDA COMO CON PTH (1-84), DURANTE 24 MESES. DATOS OBTENIDOS DE VAHLE Y COLS. (78) PARA LA TERIPARATIDA Y WILKER Y COLS. (76) PARA LA PTH (1-84)

\begin{tabular}{lccc}
\hline Dosis & Bajas & Medias & Altas \\
\hline Teriparatida $(\mu \mathrm{g} / \mathrm{kg})$ & 5 & 30 & 75 \\
PTH $(1-84)(\mu \mathrm{g} / \mathrm{kg})$ & 10 & 50 & 150 \\
\hline
\end{tabular}

Incidencia de osteosarcoma

\begin{tabular}{lccl}
\hline Machos (número) & 60 & 60 & 60 \\
Teriparatida & 3 & 21 & 31 \\
PTH (1-84) & 1 & 13 & 27 \\
Hembras (número) & 60 & 60 & 60 \\
Teriparatida & 4 & 12 & 23 \\
PTH (1-84) & 0 & 5 & 13 \\
\hline
\end{tabular}

to de riesgo oncogénico. Por lo tanto, la administración tanto de teriparatida como de PTH intacta a las dosis indicadas durante un periodo de 18 meses no produce riesgo aumentado de osteosarcoma en humanos. Aún así, en el prospecto y en la ficha técnica de ambos fármacos se hace una mención a este posible efecto secundario.

\section{¿CUÁL ES EL LUGAR DE LA PTH EN EL TRATAMIENTO DE LA OSTEOPOROSIS?}

En nuestra opinión la PTH es el tratamiento biológicamente más potente de que disponemos para el tratamiento de la osteoporosis. Tanto la teriparatida como la PTH (1-84) han sido aprobadas en nuestro país para el tratamiento de la osteoporosis postmenopáusica. Sin embargo, para situarla correctamente dentro del arsenal terapéutico, deben tenerse en cuenta diversos factores. En lo que respecta a la a la eficacia en la reducción del riesgo de fractura, no es posible efectuar comparaciones directas con otros fármacos, dada la ausencia de estudios comparativos "vis a vis", pero la reducción en el riesgo de fractura vertebral obtenida tanto por teriparatida como por la PTH intacta es similar a la descrita con otros fármacos ya aprobados con esta indicación (80-88).

En la reducción del riesgo de fracturas no vertebrales, los resultados también han sido significativos en los estudios con teriparatida mientras que la PTH 1-84 no ha demostrado reducción de este tipo de fractura, probablemente por el diseño de los estudios. Por el contrario, no se ha podido establecer un efecto beneficioso de la teriparatida ni de la PTH intacta en la fractura de cadera, a diferencia de lo descrito con otros antirresortivos $(80,86.87)$ también muy probablemente por el diseño de los estudios.

La necesidad de administrar uno u otro fármaco por medio de inyecciones subcutáneas diariamente durante 18 meses puede ser un factor que limite su utilización en algunas pacientes, sobre todo teniendo en cuenta la posibilidad de utilizar el risedronato o el alendronato una vez a la semana $(89,90)$ o el ibandronato una vez al mes (91) El perfil de segu- 
ridad de la PTH es similar al descrito con los bifosfonatos, el ranelato de estroncio y el raloxifeno $(92,93)$.

Sin embargo, el costo de la teriparatida y la PTH intacta es significativamente mayor que cualquier otro tratamiento aprobado para la osteoporosis y por todo ello, la utilización de estos fármacos deben restringirse a casos concretos, con osteoporosis grave, como por ejemplo pacientes con múltiples fracturas osteoporóticas o con un densidad mineral ósea muy baja (Tscore inferior a -3,5) (94-97) a aquellos casos en los que los pacientes no toleren otros tratamientos y tengan un elevado riesgo de fractura y finalmente podríamos también considerar aquellos casos en los que exista una mala respuesta terapéutica a otros fármacos, entendiendo como tal a la aparición de fracturas recurrentes o un descenso importante, documentado y sostenido de la densidad mineral ósea pese al tratamiento antirresortivo. Ningún tratamiento de los que disponemos en la actualidad produce una reducción total del riesgo de nuevas fracturas, por lo que el término "mala respuesta terapéutica" debe utilizarse con precaución.

Un reciente meta-análisis realizado por Vestgaard y cols. (98), pone de manifiesto que la PTH, tanto en su forma intacta como la teriparatida, producen un incremento estadísticamente significativo de la DMO y una reducción del riesgo de fractura vertebral del 64\% y de fractura no vertebral del 38\%. De acuerdo con los criterios de la medicina basada en la evidencia (99-103), los meta-análisis constituyen nivel de evidencia 1a y grado de recomendación A (Tabla IV).

Por último, y no es un dato desdeñable, otro meta-análisis ha demostrado un efecto positivo de la teriparatida en el dolor vertebral (106) dato que se está investigando en la actualidad tanto con teriparatida como con PTH 1-84, así como el posible efecto de este tratamiento en la aceleración de la curación de las fracturas.

En conclusión, un nuevo tipo de tratamiento de la osteoporosis, basado en un potente efecto osteoformador, abre nuevas perspectivas en nuestro arsenal terapéutico. Muy posiblemente su uso, aislado o en combinación con otros fármacos, posiblemente de forma secuencial, va a constituir una estrategia
TABLA IV

NIVELES DE EVIDENCIA. CEBM OXFORD (99-103)

Nivel de Tipo de estudio
evidencia

\begin{tabular}{cl}
\hline 1a & $\begin{array}{c}\text { Revisión sistemática de ensayos clínicos } \\
\text { aleatorizados, con homogeneidad }\end{array}$ \\
1b & $\begin{array}{c}\text { Ensayo clínico aleatorizado con intervalo } \\
\text { de confianza estrecho }\end{array}$ \\
1c & Práctica clínica ("todos o ninguno") \\
$2 \mathrm{a}$ & Revisión sistemática de estudios de cohor- \\
& tes, con homogeneidad \\
$2 b$ & Estudio de cohortes o ensayo clínico alea- \\
& torizado de baja calidad \\
$2 c$ & "Outcomes research", estudios ecológicos \\
$3 a$ & Revisión sistemática de estudios caso-con- \\
& trol, con homogeneidad \\
$3 b$ & Estudio caso-control \\
4 & Serie de casos o estudios de cohortes y \\
& caso-control de baja calidad \\
5 & Opinión de expertos sin valoración crítica \\
& explícita, o basados en la fisiología, \\
& "bench research" o "first principles"
\end{tabular}

habitual para la osteoporosis. Indudablemente, como ha ocurrido con el desarrollo de otros fármacos, la historia del descubrimiento y uso de la PTH ha contribuido a algunos avances decisivos en nuestro conocimiento de la fisiología del hueso y de la fisiopatología de la osteoporosis.

\section{Bibliografía}

1. NIH consensus panel. Consensus development conference: diagnosis, prophylaxis and treatment of osteoporosis. Am J Med 1993; 94: 64650.

2. NIH Consensus Development Panel on Osteoporosis Prevention, Diagnosis and Therapy. JAMA. 2001; 285: 785-95.

3. Recker RR, Barger-Lux MJ. The elusive concept of bone quality. Curr Osteoporos Rep 2004; 2: 97-100.

4. Sosa Henríquez M. Consecuencias de la osteoporosis. En: Rapado Errazti A, Díaz Curiel M, Galindo P, editores. Osteoporosis: una guía para profesionales de la Salud. Madrid: Fundación Hispana de Osteoporosis y Enfermedades Metabólicas Óseas (FHOEMO); 1997. p. 214.

5. Cummings SR, Melton LJ III. Epidemiology and outcomes of osteoporotic fractures. Lancet 2002; 359: 1761-7.

6. Sosa Henríquez M, Díez Pérez A. Osteoporosis. Concepto. Etiopatogenia. Clínica. Medicine 2006; 9 (Extr. 1): 8-14.

7. Sosa Henríquez M, Hernández Hernández D. Tratamiento de la osteoporosis. Medicine. 2006; 9 (Extr. 1): 35-9

8. Bilezikian JP, Rosen CJ. Clinical Review 123: Hot Topic. Anabolic Therapy for Osteoporosis. J Clin Endocrinol Metab 2001; 86: 957-64.

9. González Macias J, Olmos Martínez J. Fisiopatología de la osteoporosis. Medicine 2006; 9 (Extr. 1): 1-7
10. Eastell R. Pathogenesis of Postmenopausal Osteoporosis. En: Primer on the Metabolic Disease and Disorders of Mineral Metabolism, 6th Edition. Ed MJ Favus. American Society for Bone and Mineral research. Washington; 2006. p. 259-62.

11. Sambrook P, Cooper C. Osteoporosis. Lancet 2006; 367: 2010-8.

12. Black DM, Cummings SR, Karpf DB, Cauley JA, Thompson DE, Nevitt MC, et al. Randomized trial of effect of alendronate on risk of fracture in women with existing vertebral fractures. Lancet 1996; 348 : 1535-41.

13. Cummings SR, Nevitt MC, Browner WS, Stone K, Fox KM, Ensrud $\mathrm{KE}$, et al. Risk factors for hip fracture in white women. N Engl J Med 1995; 332: 767-73.

14. Dresner-Pollak R, Parker RA, Poku M, Thompson J Seibel MJ, Greenspan SL. Biochemical markers of bone turnover reflect femoral bone loss in elderly women. Calcif Tissue Int 1996; 59: 328 -33.

15. Garnero P, Sornay-Rendu E, Chapuy MC. Increased bone turnover in late postmenopausal women is a major determinant of osteoporosis. $\mathrm{J}$ Bone Miner Res 1996; 11: 337-49.

16. Garnero P, Hausherr E, Chapuy MC, Marcelli C, Grandjean H, Muller $\mathrm{C}$, et al. Markers of bone resorption predict hip fracture in elderly women: The EPIDOS study. J Bone Miner Res 1996; 11: 1531-8.

17. Delmas PD, Li Z, Cooper C. Relationship between changes in bone 
mineral density and fracture risk reduction with antiresorptive drugs: Some issues with meta-analyses. J Bone Miner Res 2004; 19: 330-7.

18. Silverberg SJ, Gartenberg F, Jacobs TP, Shane E, Siris E, Staron RB, et al. Longitudinal measurements of bone density and biochemica indices in untreated primary hyperparathyroidism. J Clin Endocrinol Metab 1995; 80: 723-8.

19. Parisien M, Cosman F, Mellish RWE, Schnitzer M, Nieves J, Silverberg SJ, et al. Bone structure in postmenopausal hyperparathyroid, osteoporotic and normal women. J Bone Miner Res 1995; 10: 1393-9.

20. Dempster DW, Parisien M, Silverberg SJ, Liang XG, Schnitzer M, Shen V, et al. On the mechanism of cancellous bone preservation in postmenopausal women with primary hyperparathyroidism. J Clin Endocrinol Metab 1999; 84: 1562-6.

21. Morley P, Whitfield JF, Willick GE. Anabolic effects of PTH on bone. Trends Endocrinol Metab 1997; 8: 225-31.

22. Hodsman AB, Bauer DC, Dempster DW, Dian L, Hanley DA, Harris ST et al. Parathyroid Hormone and teriparatida for the treatment of osteoporosis: A review of the evidence and suggested guidelines for its use. Endocrine Rev 2005; 26: 688-703.

23. Reeve J, Tregear GW. Parsons JA. Preliminary trial of low doses of human parathyroid hormone 1-34 peptide in treatment of osteoporosis Calcif Tissue Res 1976; 21 (Supl.): 469-77.

24. Reeve J, Hesp R, Williams D, Hulme P, Klenerman L, Zanelli JM, et al. Anabolic effect of low doses of a fragment of human parathyroid hormone on the skeleton in postmenopausal osteoporosis. Lancet 1976; 1 (7968): 1035-8.

25. Reeve J, Meunier PJ, Parsons JA, Bernat M, Bijvoet OL, Courpron P, et al. Anabolic effect of human parathyroid hormone fragment on trabecular bone in involutional osteoporosis: a multicentre trial. Br Med J 1980; 280: 1340-4

26. Slovik DM, Rosenthal DI, Doppelt S, Potts JT, Daly MA, Campbell JA, et al. Restoration of spine bone mass in osteoporotic men by treatment with hPTH and 1,25 vitamin D. J Bone Miner Res 1986; 1: 377 81.

27. Frost HM. Treatment of osteoporoses by manipulation of coherent bone cell populations. Clin Orthop Relat Res 1979; (143): 227-44.

28. Neer RM, Arnaud CD, Zanchetta JR, Prince R, Gaich GA, Reginster JY, et al. Effect of parathyroid hormone (1-34) on fractures and bone mineral density in postmenopausal women with osteoporosis. N Engl J Med 2001; 344; 1434-41.

29. Genant HK, Siris E, Crans GG, Desaiah D, Krege JH. Reduction in vertebral fracture risk in teriparatida-treated postmenopausal women as assessed by spinal deformity index. Bone 2005; 37: 170-4.

30. Genant HK, Halse J, Briney WG, Xie L, Glass EV, Krege JH. The effects of teriparatida on the incidence of back pain in postmenopausal women with osteoporosis. Curr Medi Res Opinión 2005; 7: 1027-34.

31. Gallagher JC, Genant HK, Crans GG, Vargas Socorro J, Krege JH. Teriparatida reduces the fracture risk associated with increasing number and severity of osteoporotic fractures. J Clin Endocrinol Metab 2005; 90: 1583-7.

32. Nevitt MC, Chen P, Dore RK, Reginster JY, Kiel DP, Zanchetta JR, et al. Reduced risk of back pain following teriparatida treatment: a metaanalysis. Osteoporos Int 2006; 17: 273-80.

33. Oglesby AK, Minshall ME, Shen W, Xie S, Silverman SL. The impact of incident vertebral and non-vertebral fragility fractures on healthrelated quality of life in established postmenopausal osteoporosis: Results from the teriparatida randomized, placebo-controlled trial in postmenopausal women. J Rheumatol 2003; 30: 1579-83.

34. Crans GG, Silverman SL, Genant HK, Glass EV,Krege JH. Association of severe vertebral fractures with reduced quality of life reduction in the incidence of severe vertebral fractures by teriparatida. Arthritis Rheumatism 2004; 50: 4028-34

35. Lindsay R, Nieves J, Formica C, Henneman E, Woelfert L, Shen V, et al. Randomised controlled study of effect of parathyroid hormone on vertebral-bone mass and fracture incidence among postmenopausal women on oestrogen with osteoporosis. Lancet 1997; 350: 550-5.

36. Body JJ, Gaich GA, Scheele WH, Kulkarni PM, Miller PD, Peretz A, et al. A randomized double-blind trial to compare the efficacy of teriparatida (recombinant human parathyroid hormone (1-34)) with alendronate in postmenopausal women with osteoporosis. J Clin Endocrinol Metab 2002; 87: 4528-35

37. Hodsman AB, Fraher LJ, Watson PH, Ostbye T, Stitt LW, Adachi JD, et al. A randomized controlled trial to compare the efficacy of cyclical parathyroid hormone versus cyclical parathyroid hormone and sequential calcitonin to improve bone mass in postmenopausal women with osteoporosis. J Clin Endocrinol Metab 1997; 82: 620-8

38. Jiang Y, Zhao JJ, Mitlak BH, Wang O, Genant HK, Eriksen EF Recombinant human parathyroid hormone (1-34) (teriparatida) improves both cortical and cancellous bone structure. J Bone Miner Res 2003, 18: 1932-41.

39. Dempster DW, Cosman F, Kurland ES, Zhou H, Nieves J, Woelfert L, et al. Effects of daily treatment with parathyroid hormone on bone microarchitecture and turnover in patients with osteoporosis: a paired biopsy study. J Bone Miner Res 2001, 16: 1846-53.

40. Burr D. Does early PTH treatment compromise bone strength? The balance between remodeling, porosity, bone mineral, and bone size. Curr Osteoporos Rep 2005, 3: 19-24.

41. Zanchetta JR, Bogado CE, Ferretti JL, Wang O, Wilson MG, Sato M, et al. Related Articles, Effects of teriparatida (recombinant human parathyroid hormone (1-34)) on cortical bone in postmenopausal women with osteoporosis. J Bone Miner Res 2003; 18: 539-43.

42. Finkelstein JS, Hayes A, Hunzelman JL, Wyland JJ, Lee H, Neer RM. The effects of parathyroid hormone, alendronate, or both in men with osteoporosis. N Engl J Med 2003; 349: 1216-26

43. Cosman F, Nieves J, Woelfert L, Formica CA, Gordon SL, Shen V, et al. Parathyroid hormone added to established hormone replacement therapy: Effects on vertebral fracture and maintenance of bone mass after parathyroid hormone withdrawal. J Bone Miner Res 2001; 16: 925-931.

44. Compston J. Recombinant Parathyroid Hormone in the Management of Osteoporosis. Calcif Tissue Int 2005; 77: 65-71.

45. Khosla S. Parathyroid hormone plus alendronate: A combination that does not add up. N Engl J Med 2003; 349-1277-9.

46. Hodsman AB, Hanley DA, Ettinger MP, Bolognese MA, Fox J, Metcalfe AJ. et al. Efficacy and safety of human parathyroid hormone-(184 ) in increasing bone mineral density in postmenopausal osteoporosis. J Clin Endocrinol Metab 2003, 88: 5212-20.

47. Greenspan SL, Bone HG, Ettinger MP, Hanley DA, Lindsay R, Zanchetta JR, et al. Effect of recombinant human parathyroid hormone (184 ) on vertebral fracture and bone mineral density in postmenopausal women with osteoporosis: A randomized trial. Ann Intern Med 2007; 146: 326-39.

48. Greenspan SL, Marriott TB, Hanley DA, Zanchetta JR, Bone HG, Ettinger MP, et al. Preventing the first vertebral fracture in postmenopausal women with low bone mass using PTH (1-84): results from the TOP study (abstract). J Bone Miner Res 2005, 20 (Supl. 1): 556.

49. Black DM, Greenspan SL, Ensrud KE, Palermo L, McGowan JA, Lang TF, et al, for the PaTH Study Investigators: The effects of parathyroid hormone and alendronate alone or in combination in postmenopausal osteoporosis. N Engl J Med 2003; 349: 1207-15.

50. Bauer DC, Garnero P, Bilezikian JP, Greenspan SL, Ensrud KE, Rosen CJ, et al, for the PTH and Alendronate (PaTH) Research Group. Short-term changes in bone turnover markers and bone mineral density response to parathyroid hormone in postmenopausal women with osteoporosis. J Clin Endocrinol Metab 2006; 91: 1370-5.

51. Fox J, Miller MA, Recker RR, Bare SP, Smith SY, Moreau I. Treatment of postmenopausal osteoporotic women with parathyroid hormone 1-84 for 18 months increases cancellous bone formation and improves cancellous architecture: A study of iliac crest biopsies using histomorphometry and micro computed tomography. J Musculoskelet Neuronal Interact 2005; 5: 356-7.

52. Antoniucci DM, Sellmeyer DE, Bilezikian JP, Palermo L, Ensrud KE Greenspan SL, et al. Elevations in Serum and Urinary Calcium with Parathyroid Hormone (1-84) with and without Alendronate for Osteoporosis. J Clin Endocrin Metab. First published ahead of print December 12, 2006 as doi: 10.1210/jc.2006-1788

53. Rittmaster RS, Bolognese RS, Ettinger MP, Hanley DA, Hodsman $\mathrm{AB}$, Kendler DL, et al. Enhancement of Bone Mass in Osteoporotic Women with Parathyroid Hormone followed by Alendronate. J Clin Endocrinol Metab 2000; 85: 2129-34.

54. Stewart AF, Horst R, Deftos LJ, Cadman EC, Lang R, Broadus AE. Biochemical evaluation of patients with cancer-associated hypercalcemia: evidence for humoral and non-humoral groups. N Engl J Med 1980; 303: 1377-83.

55. Stewart AF, Vignery A, Silvergate A, Ravin ND, LiVolsi V, Broadus $\mathrm{AE}$, et al. Quantitative bone histomorphometry in humoral hypercalcemia of malignancy: uncoupling of bone cell activity. J Clin Endocrinol Metab 1982; 55: 219-27.

56. Nakayama K, Fukumoto S, Takeda S, Takeuchi Y, Ishikawa T, Miura $\mathrm{M}$, et al. Differences in bone and vitamin D metabolism between pri- 
mary hyperparathyroidism and malignancy-associated hypercalcemia. J Clin Endocrinol Metab 1996; 81: 607-11.

57. Strewler GJ The physiology of parathyroid hormone-related protein. N Engl J Med 2000; 342: 177-85.

58. Strewler GJ, Nissenson RA. Hypercalcemia in malignancy. West J Med 1990; 153: 635-40.

59. Wysolmerski, JJ, Broadus AE. Hypercalcemia of malignancy: the central role of parathyroid hormone-related protein. Annu Rev Med 1994; 45: $189-200$

60. Grill V, Rankin W, Martin TJ. Parathyroid hormone-related protein (PTHrP) and hypercalcaemia. Eur. J. Cancer. 1998; 34: 222-9.

61. Miao D Bin He B, Jiang Y, Kobayash T, Sorocéanu MA, et al. Osteoblast-derived PTHrP is a potent endogenous bone anabolic agent that modifies the therapeutic efficacy of administered PTH 1-34. J Clin Invest 2005; 115: 240-11.

62. Abou-Samra AB, Juppner H, Force T, Freeman MW, Kong XF, Schipani E, et al. Expression cloning of a common receptor for parathyroid hormone and parathyroid hormone-related peptide from rat osteoblastlike cells: a single receptor stimulates intracellular accumulation of both cAMP and inositol trisphosphates and increases intracellular free calcium. Proc Natl Acad Sci USA 1992; 89: 2732-6.

63. Walsh CA, Birch MA, Fraser WD, Lawton R, Dorgan J, Walsh S, et al. Expression and secretion of parathyroid hormone-related protein by human bone-derived cells in vitro: effects of glucocorticoids. J Bone Miner Res 1995; 10: 17-25.

64. Horwitz MJ, Tedesco MB, Sereika SM, Hollis, BW, García-Ocaña A, Stewart AF. Direct Comparison of sustained infusion of human parathyroid hormone-related protein-(1-36) (HPTHRP-(1-36)) versus HPTH-(1-34) on serum calcium, plasma 1,25-dihydroxyvitamin D concentrations, and fractional calcium excretion in healthy human volunteers. J Clin Endocrinol Metab 2003; 88: 1063-9.

65. Horwitz MJ, Tedesco MB, Gundberg C, García-Ocaña A, Stewart AF. Short-term, high-dose parathyroid hormone-related protein as a skeletal anabolic agent for the treatment of postmenopausal osteoporosis. J Clin Endocrinol Metab 2003; 88: 569-75.

66. Kurland ES, Cosman F, McMahon DJ, Rosen CJ, Lindsay R, Bilezikian JP. Parathyroid hormone as a therapy for idiopathic osteoporosis in men: effects on bone mineral density and bone markers. J Clin Endocrinol Metab 2000; 85: 3069-76.

67. Orwoll ES, Scheele WH, Paul S, Adami S, Syversen U, Díez-Perez A, et al. The effect of teriparatida (human parathyroid hormone (1-34)) therapy on bone density in men with osteoporosis. J Bone Miner Res 2003; 18: 9-17.

68. Kaufman JM, Orwoll E, Goemaere S, San Martin J, Hossain A, Dalsky GP, et al. Teriparatida effects on vertebral fractures and bone mineral density in men with osteoporosis: treatment and discontinuation of therapy. Osteoporosis Int 2005; 16: 510-6.

69. Lindsay R, Scheele W, Neer N, Pohl G, Adami S, Mautalen C, et al. Sustained vertebral fracture risk reduction alter withdrawal of teriparatida in postmenopausal women with osteoporosis. Arch Intern Med 2004; 164: 2024-30.

70. Rehman Q, Lang TF, Arnaud CD, Modin GW, Lane NE. Daily treatment with parathyroid hormone is associated with an increase in vertebral cross-sectional area in postmenopausal women with glucocorticoid-induced osteoporosis. Osteoporos Int 2003; 14: 77-81.

71. Hurley M, Yao W, Lane NE. Changes in serum fibroblast growth factor 2 in patients with glucocorticoid-induced osteoporosis treated with human parathyroid hormone (1-34). Osteoporos Int 2005; 16: 2080-4.

72. Lane NE, Sánchez S, Modin GW, Genant HK, Pierini E, Arnaud CD. Parathyroid hormone treatment can reverse corticosteroid-induced osteoporosis. Results of a randomized controlled clinical trial. J Clin Invest $1998 ; 102$ : 1627-33.

73. Lane NE, Sanchez S, Genant HK, Jenkins DK, Arnaud CD. Short term increases in bone turnover markers predict parathyroid hormone-induced spinal bone mineral density gains in postmenopausal women with glucocorticoid-induced osteoporosis. Osteoporos Int 2000; 11: 43442.

74. Armen H Tashjian Jr AH, Gagel RF. Teriparatida (human PTH(134)): 2.5 years of experience on the use and safety of the drug for the treatment of osteoporosis. J Bone Miner Res 2006; 21: 354-65.

75. Vahle JL, Sato M, Long GG, Young JK, Francis PC, Engelhardt JA, et al. Changes in rats given daily subcutaneous injections of recombinant human parathyroid hormone (1-34) for 2 years and relevance to human safety. Toxicol Pathol 2002; 30: 312-21.

76. Wilker CE, Jolette J, Smith SY, Doyle N, Hardisty JF, Metcalf AJ, et al. A no observable carcinogenic effect dose-level identified in Fisher 344 rats following daily treatment with PTH (1-84) for 2 years: Role of the C-terminal PTH receptor. J Bone Miner Res 2004; 19: S1; S98.

77. Vahle JL, Sato M, Long GG, Young JK, Francis PC, Engelhardt JA, et al. Skeletal changes in rats given daily subcutaneous injections of recombinant human parathyroid hormone (1-34) for 2 years and relevance to human safety. Toxicol Pathol 2002; 30: 312-21.

78. Vahle JL, Long GG, Sandusky G, Westmore M, Ma YL, Sato M. Bone neoplasms in F344 rats given teriparatida $(\operatorname{rhPTH}(1-34))$ are dependent on duration of treatment and dose. Toxicol Pathol 2004; 32 : 426-38.

79. Tashjian AH, Gagel RF. Teriparatida (Human PTH(1-34)): 2.5 years of experience on the use and safety of the drug for the treatment of osteoporosis. J Bone Miner Res 2006; 21: 354-65.

80. Black DM, Cummings SR, Karpf DB, Cauley JA, Thompson DE, Nevitt MC, et al. Randomised trial of effect of alendronate on riskof fracture in women with existing vertebral fractures. Lancet 1996; 348: $1535-41$.

81. Ettinger B, Black DM, Mitlak BH, Knickerbocker RK, Nickelsen T, Genant HK, et al. Reduction of vertebral fracture riskin postmenopausal women with osteoporosis treated with raloxifene: results from a 3year randomized clinical trial. JAMA 1999; 282: 637-45

82. Harris ST, Watts NB, Genant HK, McKeever CD, Hangartner T, Keller M, et al. Effects of risedronate treatment on vertebral and nonvertebral fractures in women with postmenopausal osteoporosis. A randomized controlled trial. JAMA 1999; 282: 1344-52.

83. Reginster J-Y, Minne HW, Sorensen OH, Hooper M, Roux C, Brandi $\mathrm{ML}$, et al. Randomized trial of the effects of risedronate on vertebral fractures in women with established postmenopausal osteoporosis. Osteoporos Int 2000; 11: 83-91.

84. Chesnut CH, Silverman S, Andriano K, Genant HK, Gimona A, Harris $\mathrm{S}$, et al. A randomised trial of nasal spray salmon calcitonin in postmenopausal women with established osteoporosis: the Prevent Recurrence of Osteoporotic Fractures Study. Am J Med 2000; 109: 267-76.

85. Meunier PJ, Roux C, Seeman E, Ortolani S, Badurski JE, Spector TD, et al. The effects of strontium ranelate on the riskof vertebral fracture in women with postmenopausal osteoporosis. N Engl J Med 2004; 350: 459-68.

86. McClung MR, Geusens P, Miller PD, Zippel H, Benson WG, Roux C, et al. Effect of risedronate on the risk of hip fracture in elderly women. N Engl J Med 2001; 344: 333-40.

87. Reginster JY, Seeman E, DeVernejoul MC, Adami S, Compston J, Phenekos C, et al. Strontium ranelate reduces the risk of nonvertebral fractures in postmenopausal women with osteoporosis: Treatment of peritoneal osteoporosis. TROPOS) study. J Clin Endocrinol Metab 2005; 90: 2816-XXX

88. Chesnut CH III, Skag A, Christiansen C, Recker R, Stakkestad JA, Hoiseth A, et al. Effects of oral ibandronate administered daily or intermittently on fracture risk in postmenopausal osteoporosis. J Bone Miner Res 2004; 19: 1241-9.

89. Schnitzer T, Bone HG, Crepaldi G, Adami S, McClung M, Kiel D, et al. Therapeutic equivalence of alendronate $70 \mathrm{mg}$ once-weekly and alendronate $10 \mathrm{mg}$ daily in the treatment of osteoporosis. Alendronate Once-Weekly Study Group. Aging (Milano) 2000; 12: 1-12.

90. Ilter E, Karalok H, Tufekci EC, Batur O. Efficacy and acceptability of risedronate $5 \mathrm{mg}$ daily compared with $35 \mathrm{mg}$ once weekly for the treatment of postmenopausal osteoporosis. Climacteric 2006; 9: 129-34

91. Millar PD, McClung MR, Macovei L, Stakkestad JA, Luckey M, Bonvoisin B., et al. Monthly oral ibandronate therapy on postmenopausal osteoporosis: 1-year results from the MOBILE Study. J Bone Miner Res 2005; 20: 1315-22.

92. Delmas PD, Ensrud KE, Adachi JD, Harper KD, Sarkar S, Gennari C, et al. Efficacy of raloxifene on vertebral fracture risk reduction in postmenopausal women with osteoporosis: four-year results from a randomized clinical trial. J Clin Endocrinol Metab 2002; 87: 3609-17.

93. Meunier PJ, Roux C, Seeman E, Ortolani S, Badurski JE, Spector TD, et al. The effects of strontium ranelate on the risk of vertebral fracture in women with postmenopausal osteoporosis. N Engl J Med 2004; 350: 459-68.

94. Deal C. The Use of Intermittent Human Parathyroid Hormone As a Treatment for Osteoporosis. Current Rheumatology Reports 2004, 6: 49-58.

95. Cosman F. Anabolic Therapy for Osteoporosis: Parathyroid Hormone. Current Osteoporosis Reports 2005, 3: 143-9. 
96. Girotra M,Rubin MR, Bilezikian JP. Anabolic Skeletal Therapy for Osteoporosis. Arq Bras Endocrinol Metab 2006; 50: 745-54.

97. Quesada JM. Agentes anabólicos óseos para el tratamiento de osteoporosis. Endocrinol Nutr 2004; 51: 512-23.

98. Vestergaard P, Jorgensen NR, Mosekilde L, Schwarz P. Effects of parathyroid hormone alone or in combination with antiresorptive therapy on bone mineral density and fracture risk - a meta-analysis. Osteoporos Int 2007; 18: 45-7.

99. Evidence-Based Medicine Working Group. Evidence-based medicine. A new approach to teaching the practice of medicine. JAMA 1992; 268: 2420-5

100. Sackett DL, Rosenberg WMC, Gary JAM, Haynes RB, Richardson WS. Evidence based medicine: what is it and what it isn't. BMJ 1996; 312: 71-2.

101. Guerra Romero L. La medicina basada en la evidencia: un intento de acercar la ciencia al arte de la práctica clínica. Med Clin (Barc) 1996; 107: $377-82$
102. Jovell AJ, Navarro-Rubio MD. Evaluación de la evidencia científica. Med Clin (Barc) 1995; 105: 740-3.

103. Guyatt GH, Sackett DL, Sinclair JC, Hayward R, Cook DJ, Cook RJ. Users' Guides to the Medical Literature: IX. A method for grading health care recommendations. JAMA 1995; 274: 1800-4.

104. Deal C, Omizo M, Schwartz EN, Eriksen EF, Cantor P, Wang J, et al. Combination teriparatida and raloxifene therapy for postmenopausal osteoporosis: results from a 6-month double-blind placebo-controlled trial. J Bone Miner Res 2005; 20: 1905-11.

105. Harper KD, Krege JH, Marcus R, Mitlak BH. Osteosarcoma and teriparatida? J Bone Miner Res. 2007; 22: 334

106. Nevitt MC, Chen P, Dore RK, Reginster JY, Kiel DP, Zanchetta JR, et al. Reduced risk of back pain following teriparatida treatment: a metaanalysis. Osteoporos Int. 2006; 17: 273-80.

107. Black DM, Bilezikian JP, Ensrud KE, Greenspan SL, Palermo L, et al. One Year of Alendronate after One Year of Parathyroid Hormone (184) for Osteoporosis. N Engl J Med 2005; 353: 555-65. 